PREPARED FOR THE U.S. DEPARTMENT OF ENERGY, UNDER CONTRACT DE-AC02-76CH03073

PPPL-3786

PPPL-3786

UC-70

Infernal Fishbone Mode

by

Ya.I. Kolesnichenko, V.S. Marchenko, and R.B. White

February 2003

NM|

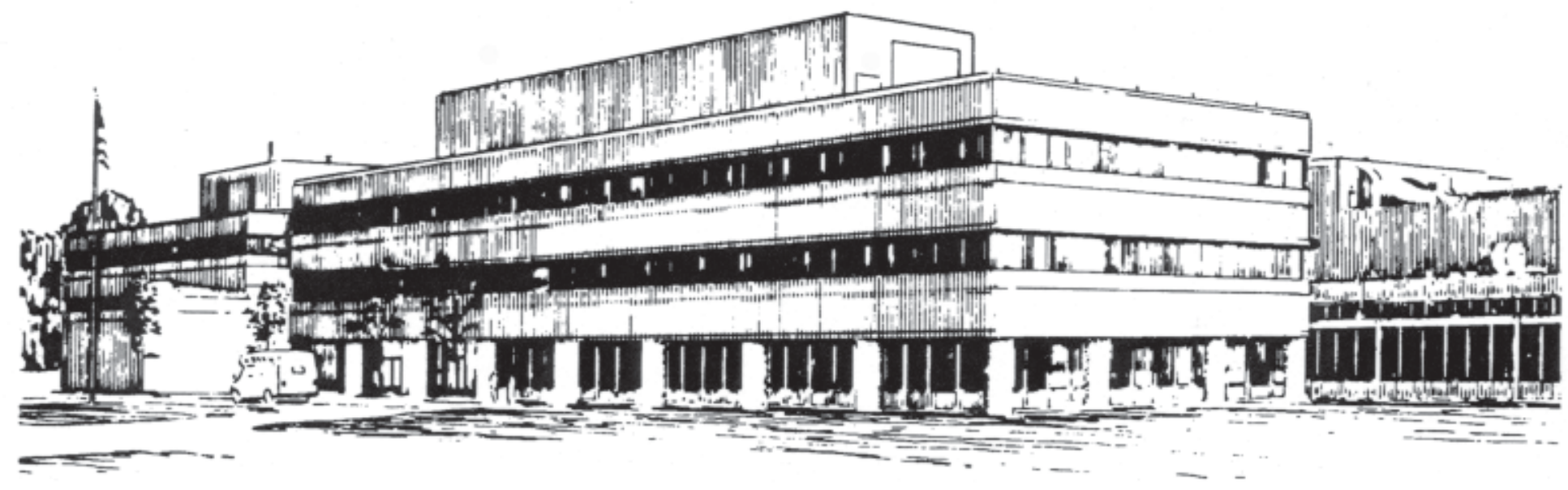

PRINCETON PLASMA PHYSICS LABORATORY PRINCETON UNIVERSITY, PRINCETON, NEW JERSEY 


\section{PPPL Reports Disclaimer}

This report was prepared as an account of work sponsored by an agency of the United States Government. Neither the United States Government nor any agency thereof, nor any of their employees, makes any warranty, express or implied, or assumes any legal liability or responsibility for the accuracy, completeness, or usefulness of any information, apparatus, product, or process disclosed, or represents that its use would not infringe privately owned rights. Reference herein to any specific commercial product, process, or service by trade name, trademark, manufacturer, or otherwise, does not necessarily constitute or imply its endorsement, recommendation, or favoring by the United States Government or any agency thereof. The views and opinions of authors expressed herein do not necessarily state or reflect those of the United States Government or any agency thereof.

\section{Availability}

This report is posted on the U.S. Department of Energy's Princeton Plasma Physics Laboratory Publications and Reports web site in Fiscal Year 2003. The home page for PPPL Reports and Publications is: http://www.pppl.gov/pub_report/

DOE and DOE Contractors can obtain copies of this report from:

U.S. Department of Energy

Office of Scientific and Technical Information

DOE Technical Information Services (DTIS)

P.O. Box 62

Oak Ridge, TN 37831

Telephone: (865) 576-8401

Fax: (865) 576-5728

Email: reports@adonis.osti.gov

This report is available to the general public from:

National Technical Information Service

U.S. Department of Commerce

5285 Port Royal Road

Springfield, VA 22161

Telephone: $1-800-553-6847$ or

(703) $605-6000$

Fax: (703) 321-8547

Internet: http://www.ntis.gov/ordering.htm 


\title{
Infernal fishbone mode
}

\author{
Ya.I.Kolesnichenko', V.S.Marchenkoํㅛ R.B. White ${ }^{2}$ \\ 1 Institute for Nuclear Research, \\ National Academy of Sciences of Ukraine, Kyiv, 03680, Ukraine \\ 2 Princeton Plasma Physics Laboratory, P.O. Box 451, \\ Princeton, New Jersey, 08543, USA
}

(February 5, 2003)

\begin{abstract}
A new kind of fishbone instability associated with circulating energetic ions is predicted. The considered instability is essentially the energetic particle mode; it is characterized by $m / n \neq 1$ ( $m$ and $n$ are the poloidal and toroidal mode numbers, respectively); the mode is localized inside the flux surface where the safety factor $(q)$ is $q_{*}=m / n$, its amplitude being maximum near $q_{*}$; the instability arises in plasmas with small shear inside the $q_{*}$ surface and $q(0)>1$. A possibility to explain recent experimental observations of the $m=2$ fishbone oscillations accompanied by strong changes of the neutron emission during tangential neutral beam injection in the National Spherical Torus Experiment [M.Ono et al., Nucl. Fusion 40, 557 (2000)] is shown.
\end{abstract}


The fishbone instability belongs to most interesting and important phenomena in toroidal plasmas with energetic ions. ${ }^{-4}$ It can be very dangerous, leading to expulsion of a considerable fraction (up to 50\%) of energetic ions. ${ }^{5}$ But, on the other hand, sometimes it is useful, being a trigger of formation of the internal transport barrier. ${ }^{6}$ Typically, the fishbone instability is associated with an $m=n=$ 1 perturbation ( $m$ and $n$ are the poloidal and toroidal mode numbers, respectively) in the plasma core. Theory predicts that features of the fishbone mode in spherical tori (ST) differs from those in tokamaks. In particular, the high $\beta$ (the ratio of plasma pressure to the magnetic field pressure) tends to stabilize the known fishbone instabilities (which can be associated with trapped or circulating energetic ions) $)^{7,8}$ but, on the other hand, a new new kind of the fishbone mode ("bouncefrequency fishbones") can arise in moderate- $\beta$ shots of STs. ${ }^{9}$ A theory developed in Ref. $^{9}$ seems to explain recent experimental observations of the $m=2$ fishbones in the NSTX spherical torus, shot \#106218. ${ }^{9}$ The mentioned theory suggests that the instability is caused by the trapped energetic ion population. The latter appears in the NSTX shot \#106218 mainly due to pitch-angle scattering of partly slowed down injected ions having the energy $\mathcal{E} \ll \mathcal{E}_{0}$, where $\mathcal{E}_{0}=80 \mathrm{keV}$ is the injection energy. The theoretical conclusion in Ref. ${ }^{9}$ that the energy of particles driving the instability is relatively small is consistent with the fact that fishbones only weakly affect the neutron yield in the mentioned shot (the neutron production in NSTX is predominantly from beam-plasma reactions). However, recently results of other observations of $m=2$ fishbones in NSTX were reported, showing strong influence of the fishbone instability on the neutron yield. ${ }^{10}$ This indicate that particles with the energy $\mathcal{E} \sim 80 \mathrm{keV}$ are involved to the process, which motivated the present work. In this Letter, we show that energetic circulating particles can result in a new $m \neq 1$ instability in plasmas with a wide shearless plasma core characterized by the safety factor, $q$, close to but less than $m / n$. The physics of the considered instability differs from the known circulating-particle-induced instability of the $m=n=1$ mode $^{11,12,8}$ because of different radial mode structure. We will refer to a new 
instability as "infernal fishbones" because the infernal instability is known to be driven by pressure gradient in low-shear plasmas (in our case the instability is driven by the pressure gradient of the energetic ions, although the velocity anisotropy of the energetic ions can be a destabilizing factor, too).$^{13-15}$

We proceed from the following vorticity equation: ${ }^{16}$

$$
\vec{B} \cdot \nabla\left[\frac{1}{B^{2}} \nabla_{\perp}^{2}(\vec{A} \cdot \vec{B})\right]-\frac{i \omega c}{v_{A}^{2}} \nabla_{\perp}^{2} \Phi+\frac{4 \pi}{B^{2}} \vec{\kappa} \times \vec{B} \cdot \nabla_{\perp}\left(2 \delta p_{c}+\delta p_{\| \alpha}+\delta p_{\perp \alpha}\right)=0
$$

where $B$ is the magnetic field strength, $\vec{A}$ and $\Phi$ are the mode vector potential and electrostatic potential, respectively, $\vec{\kappa}=\vec{b} \cdot \nabla \vec{b}$ with $\vec{b}=\vec{B} / B$ is the magnetic field line curvature, $v_{A}$ is the Alfvén velocity, $\delta p$ is the perturbed pressure, the subscript $c$ is relevant to the bulk plasma, and subscript $\alpha$ labels the energetic ion magnitudes, $\nabla_{\perp}=\nabla-\vec{b}(\vec{b} \cdot \nabla)$. Using a standard technique, Eq. (1) can be reduced to a single second order differential equation for the $m>1$ radial displacement given by

$$
\begin{aligned}
\frac{1}{r} \frac{d}{d r} r^{3}\left[\frac{\omega^{2}}{v_{A}^{2}}\left(1+2 q^{2}\right)-k_{\|}^{2}\right] \frac{d \xi}{d r}-\left(m^{2}-1\right) & {\left[\frac{\omega^{2}}{v_{A}^{2}}\left(1+2 q^{2}\right)-k_{\|}^{2}\right] \xi } \\
& =-[\hat{g}(r, \xi)+\hat{h}(r, \xi)],
\end{aligned}
$$

where $k_{\|} \equiv(m-n q) / q R \simeq$ const for $r<r_{0}$ with $q\left(r_{0}\right)=m / n, m-n q \ll$ $m$, the term $\left(1+2 q^{2}\right)$ is an enhancement of the inertia due to toroidal effects ${ }^{17}$, $\hat{g}(r, \xi)$ represents the bulk plasma fluid-potential energy including the stabilizing Mercier term, the destabilizing contribution of toroidal coupling ${ }^{15}$ and the beam fluid contribution, and $\hat{h}(r, \xi)$ represents the kinetic contribution given by ${ }^{18}$

$$
\begin{gathered}
\hat{h}(r, \xi)=\frac{2 m r}{B_{0}^{2}} \int_{-\pi}^{\pi} \exp (i \omega t+i n \phi-i m \theta) \overrightarrow{b_{0}} \times \vec{\kappa} \cdot \nabla\left(\delta p_{\perp \alpha}^{k}+\delta p_{\| \alpha}^{k}\right) d \theta, \\
\left(\begin{array}{c}
\delta p_{\perp \alpha}^{k} \\
\delta p_{\| \alpha}^{k}
\end{array}\right) \equiv i m_{\alpha}^{2} \int d^{3} v\left(\begin{array}{c}
\frac{v_{\perp}^{2}}{2} \\
v_{\|}^{2}
\end{array}\right)\left[\left(\omega-n \omega_{* \alpha}\right) \frac{\partial F_{j}}{\partial \mathcal{E}} \int_{-\infty}^{t}\left(\frac{v_{\perp}^{2}}{2}+v_{\|}^{2}\right) \vec{\kappa} \cdot \overrightarrow{\xi_{\perp}} d t^{\prime}\right],
\end{gathered}
$$

where $\omega_{* \alpha} \equiv\left(\partial F_{\alpha} / \partial P_{\phi}\right)\left(\partial F_{\alpha} / \partial \mathcal{E}\right)^{-1}$. Treating the right-hand side (RHS) of Eq. (2) as a perturbation and assuming that $k_{\|}(r) v_{A} \simeq$ const in the shear-free core we obtain for the lowest order eigenmode in the "outer" region, $r<r_{0}$, as 


$$
\xi_{0}(r)=\xi_{00}\left(\frac{r}{r_{0}}\right)^{m-1} H\left(r_{0}-r\right)
$$

For $r>r_{0}$ the mode amplitude should decrease rapidly (on the scale $x \equiv(r-$ $\left.r_{0}\right) / r_{0} \sim \omega / \omega_{A} \ll 1$ with $\left.\omega_{A}=v_{A} / R\right)$ in order to minimize the continuum damping at the local Alfvén resonance $\omega=k_{\|} v_{A} /\left(1+2 q^{2}\right)$. An approximate eigenmode equation in the large-shear layer around $r=r_{0}$ can be obtained retaining only the higher order derivative term in Eq. (2):

$$
\frac{d}{d r}\left\{\left[\frac{\omega^{2}}{v_{A}^{2}}\left(1+2 q^{2}\right)-k_{\|}^{2}\right]\right\} \frac{d \xi}{d r}=0 .
$$

Integrating Eq. (6) once with the integration constant chosen to match with the solution given by Eq. (5) outside the layer, we obtain $d \xi / d r$. The latter we put in the following expression for the sum of the kinetic and potential energies in the layer, $\mathrm{I}:{ }^{19}$

$$
I=-2 \pi^{2} \frac{R_{0}}{m^{2}} n_{0} m_{i} \int_{\text {layer }} r^{3}\left(\frac{d \xi}{d r}\right)^{2}\left[\omega^{2}\left(1+2 q^{2}\right)-k_{\|}^{2} v_{A}^{2}\right] d r
$$

where $n_{0} m_{i}$ is the plasma mass density. As a result, we have:

$$
\begin{aligned}
& I=\pi r_{0}^{2} \frac{B_{0}^{2}}{2 R_{0}} \xi_{00}^{2}\left\{\int_{-\infty}^{\infty}\left[\left(-i \frac{\omega}{\omega_{A}}\right)^{2} \frac{1+2 q^{2}}{m^{2}}+\frac{s_{0}^{2} x^{2}}{q^{2}}\right]^{-1} d x\right\}^{-1}= \\
& -i \frac{\omega}{v_{A}} \frac{B_{0}^{2}}{2} r_{0}^{2} \xi_{00}^{2} \frac{s_{0}}{m^{2}} \sqrt{n^{2}+2 m^{2}},
\end{aligned}
$$

where $s_{0}$ is the magnetic shear at the $q_{*}=m / n$ surface. Summing Eq. (8) with potential energy in the small-shear region calculated using eigenfunction given by Eq. (5) results in the following generic eigenvalue equation:

$$
-i \frac{\omega}{\hat{\omega}_{A}}+\delta \hat{W}_{f}+\delta \hat{W}_{k}=0
$$

where

$$
\begin{gathered}
\hat{\omega}_{A}=\frac{v_{A}}{R s_{0}} \frac{m^{2}}{\sqrt{n^{2}+2 m^{2}}}, \\
\delta \hat{W}_{k} \equiv \frac{R}{r_{0}^{2} \xi_{00}^{2} B_{0}^{2}} \int{\overrightarrow{\xi_{\perp}^{*}}}^{2} \nabla \delta \Pi_{\alpha}^{k} d^{3} r \\
=-\frac{2 \pi^{2} R m_{\alpha}}{\omega_{c \alpha} r_{0}^{2} \xi_{00}^{2} B_{0}^{2}} \sum_{\sigma} \int v^{3} d v \int d P_{\phi} \int d \Lambda \tau_{b} \frac{\partial F_{\alpha}}{\partial \mathcal{E}} \frac{\omega \omega_{* \alpha}}{\omega-k_{\|} v_{\|}} \\
\times\left|\left\langle\left(\frac{v_{\perp}^{2}}{2}+v_{\|}^{2}\right) \overrightarrow{\xi_{0}} \cdot \vec{\kappa} \exp \left[i\left(\omega-k_{\|} v_{\|}\right) t\right]\right\rangle\right|^{2},
\end{gathered}
$$


$\delta \Pi_{\alpha}^{k}=\delta p_{\perp \alpha}^{k} \hat{I}+\left(\delta p_{\| \alpha}^{k}-\delta p_{\perp \alpha}^{k}\right) \overrightarrow{b \vec{b}}$ is the pressure tensor with $\delta p_{\|, \perp \alpha}^{k}$ given by Eq. (4), $\sigma \equiv v_{\|} /\left|v_{\|}\right|, \Lambda \equiv \mu B_{0} / \mathcal{E}, \tau_{b}$ is the particle transit time, $\hat{I}$ is the identity tensor and $\langle\ldots\rangle$ denotes the orbit averaging. Note that when obtaining Eq. (11), we have used the relation $[\nabla \cdot(\overrightarrow{b b})]_{\perp}=\vec{\kappa}$ and an expression for the phase space volume element ${ }^{20}$

$$
d^{3} r d^{3} v=\sum_{\sigma} \frac{2 \pi^{2} v^{3}}{m_{\alpha} \omega_{c \alpha}} d \tau d v d \Lambda d P_{\phi}
$$

where $\tau$ is the time along the orbit, and $P_{\phi}$ is the canonical angular momentum. Below we omit the terms odd in $\theta$ in $\vec{\xi} \cdot \vec{\kappa}$ in the integrand of Eq. (11), which yields

$$
\overrightarrow{\xi_{0}} \cdot \vec{\kappa}=-\frac{\xi_{00}}{R_{0}}\left(\frac{r(t)}{r_{0}}\right)^{m-1} H\left(r_{0}-r(t)\right) \cos [\theta(t)] \exp [i(m \theta(t)-n \phi(t)-\omega t)]
$$

where

$$
\begin{array}{r}
r(t)=\bar{r}-\Delta_{\alpha} \cos [\theta(t)], \quad \Delta_{\alpha}=\frac{q(\bar{r})}{v_{\|} \omega_{c \alpha}}\left(\frac{v_{\perp}^{2}}{2}+v_{\|}^{2}\right), \\
\theta(t)=\frac{v_{\|}}{q(\bar{r}) R} t, \quad \phi(t)=\frac{v_{\|}}{R} t,
\end{array}
$$

$\omega_{c \alpha}$ is the fast ion gyrofrequency, and $\bar{r}$ is a constant of motion $[\bar{r}=r(\theta=\pi / 2)]$.

The considered mode does not have the Alfvén resonance in the small-shear core. Nevertheless, Cherenkov resonance with circulating particles in this region is possible when the energetic ions are highly super-Alfvénic (which is the case in NSTX experiments with neutral beam injection).

Below we assume that the energetic-ion population consists of well-circulating particles with the equilibrium distribution function given by

$$
F_{\alpha}=\frac{\sqrt{2} m_{\alpha}^{3 / 2}}{\pi \varepsilon_{\alpha}} p_{\alpha}(\bar{r}) H\left(\mathcal{E}_{\alpha}-\mathcal{E}\right) \mathcal{E}^{-3 / 2} \delta(\Lambda)
$$

where $p_{\alpha}(\bar{r})$ is approximately the beam particle pressure, $H(x)$ is the Heavyside function. Substituting Eqs. (13)-(15) into Eq. (11), performing orbit averaging and velocity space integration, we finally obtain a dispersion relation in the form :

$$
-i \frac{\Omega}{\Omega_{A}}+\delta \hat{W}_{f}+C\left[1+\frac{5}{4} \Omega+\frac{5}{3} \Omega^{2}+\frac{5}{2} \Omega^{3}+5 \Omega^{4}+5 \Omega^{5} \ln \left(1-\frac{1}{\Omega}\right)\right]=0,
$$


where $\Omega \equiv \omega / \bar{k}_{\|} v_{\alpha}, \Omega_{A} \equiv \hat{\omega}_{A} / \bar{k}_{\|} v_{\alpha}, \bar{k}_{\|}$is some average value of $k_{\|}(r)$ in the smallshear core,

$$
C=-\frac{m^{4}(m-1)^{2}}{5 \pi n^{3}} \frac{R \rho_{\alpha}^{3}}{r_{0}^{4}} \int_{0}^{r_{0}}\left(\frac{r}{r_{0}}\right)^{2(m-2)} \frac{d \beta_{\alpha}}{d r} d r
$$

$\rho_{\alpha}$ is the Larmor radius at injection energy, and $\omega \ll \omega_{* \alpha}$ has been assumed. Note that, in contrast with a theory of the $m=n=1$ fishbone instability, ${ }^{12}$ we neglected a contribution from particles crossing the $q_{*}$ surface, assuming poor population of energetic ions at this surface. In our case of $m / n \neq 1$, the fast ion drive is dominated by the particles deposited in the shear-free core due to radial variation of a poloidal electric field of the mode, whereas almost no energy exhange occurs between these particles lying inside the $q=1$ surface and the $m=n=1$ rigid shift perturbation.

Let us consider a specific example relevant to NSTX observations of the $m / n=$ 2/1 fishbone instability strongly affecting the neutron yield. We take $s_{0}=0.6$, $\beta_{\alpha}(r)=\beta_{\alpha 0}\left(1-r^{2} / a^{2}\right)^{\nu}$ with $\nu \gg 1$, in which case $\int_{0}^{r_{0}}\left(d \beta_{\alpha} / d r\right) d r \simeq-\beta_{\alpha 0}$, and $\rho_{\alpha}=20 \mathrm{~cm}, r_{0}=40 \mathrm{~cm}, R=100 \mathrm{~cm}$, so that $R \rho_{\alpha}^{3} / r_{0}^{4}=0.31$. Then, assuming that in the absence of the energetic ions a plasma is marginally stable $\left(\delta \hat{W}_{f} \simeq 0\right)$, we obtain from Eq. (16):

$$
\Omega \simeq 0.93, \quad \beta_{\alpha 0}^{c r} \simeq 3.6 \%
$$

where $\Omega$ and $\beta_{\alpha 0}^{c r}$ are the frequency and the threshold on-axis fast ion beta at the mode onset, respectively. For the shot with $q_{0}=1.7$, we obtain $f \simeq 44.4 k \mathrm{~Hz}$ assuming $\overline{k_{\|}} R \simeq 0.1$, which compares favorably with observed initial fishbone frequency in the plasma frame $f \simeq 45 k H z$ and volume averaged beam ion beta $\left\langle\beta_{\alpha}\right\rangle \simeq 2 \%$

In summary, we have shown for the first time that the $m / n>1$ mode in plasmas with monotonic $q(r)>1$ can be destabilized by the interaction with energetic circulating ions. The istability is an energetic particle mode, the Cherenkov resonance being responsible for the interaction between the energetic ions and the perturbation. In contrast to the conventional $m=n=1$ circulating-ion-driven 
fishbone instability, the considered instability is caused mainly by particles with orbits inside the flux surface with the radius $r_{0}$ where the safety factor is $q_{*}=m / n$ (rather than by the particles crossing the $q_{*}$ surface). The strong energy exchange between these particles and the perturbation takes place due to finite orbit width of the energetic ions and a specific radial mode structure, $\delta E_{\theta} \propto r^{m-1}$ at $r<r_{0}$. Both the mode frequency and critical fast ion pressure are in reasonable agreement with experimental observations of bursting $m=2$ fishbone oscillations accompanied by strong changes of the neutron yield in the NSTX spherical torus.

\section{Acknowledgements.}

The research described in this publication was made possible in part by Award No. UP2-2419-KV-02 of the US Civilian Research \& Development Foundation and the Government of Ukraine, and U.S. Department of Energy Grant DE-FG0394ER54271. 


\section{REFERENCES}

${ }^{1}$ PDX Group, Princeton Plasma Physics Lab, Phys. Rev. Lett. 50, 891 (1983).

${ }^{2}$ R.B. White, R.J. Goldston, K. McGuire, A.H. Boozer, D.A. Monticello, and W. Park, Phys. Fluids 26, 2958 (1983).

${ }^{3}$ L. Chen, R.B. White, and M.N. Rosenbluth, Phys. Rev. Lett. 52, 1122 (1984).

${ }^{4}$ B. Coppi and F. Porcelli, Phys. Rev. Lett. 57, 2272 (1986).

${ }^{5}$ W.W. Heidbrink, G. Sager, Nucl. Fusion 30, 1015 (1990).

${ }^{6}$ S. Günter et al., in Proceedings of the 18th Fusion Energy Conference, Sorrento, Italy (2000), paper P1.006.

${ }^{7}$ Ya.I. Kolesnichenko, V.V. Lutsenko, V.S. Marchenko, Phys. Rev. Lett. 82, 3260 (1999).

${ }^{8}$ Ya.I. Kolesnichenko, V.S. Marchenko, R.B. White, Phys. Plasmas 8, 3143 (2001).

${ }^{9}$ E.Fredrickson, R.White, and L.Chen, "Bounce frequency fishbones in NSTX", preprint PPPL ???

${ }^{10}$ D.S. Darrow, S.S. Medley, A.L. Roquemore et al., "Measurements of prompt and MHD-induced fast ion loss from National Spherical Torus plasmas", the poster EX/P2-01 in the 19th IAEA Fusion Energy Conference, Lyon, France (2002).

${ }^{11}$ W.W. Heidbrink, K. Bol, D. Buchanauer et al., Phys. Rev. Lett. 57, 835 (1986).

${ }^{12}$ R. Betti and J.P. Freidberg, Phys. Rev. Lett. 70, 3428 (1993).

${ }^{13}$ L.E. Zakharov, Nucl. Fusion 18, 335 (1978).

${ }^{14}$ J. Manickam, N. Pomphrey, and A.M.M. Todd, Nucl. Fusion 27, 1461 (1987).

${ }^{15}$ L.A. Charlton, R.J. Hastie, and T.C. Hender, Phys. Fluids B1, 798 (1989).

${ }^{16}$ M.S. Chance, R.L. Dewar, E.A. Frieman, A.H. Glasser, J.M. Greene, R.C. 
Grimm, S.C. Jardin, J.L. Johnson, J. Manickam, M. Okabayashi, and A.M.M. Todd, in Plasma Physics and Controlled Thermonuclear Fusion Research (IAEA, Vienna, 1978), Vol.I, p.677.

${ }^{17}$ A.H. Glasser, J.M. Greene, and J.L. Johnson, Phys. Fluids 18, 875 (1975).

${ }^{18}$ B. Coppi, S. Migliuolo, and F. Porcelli, Phys. Fluids 31, 1630 (1988).

${ }^{19}$ C.G. Gimblett, R.J. Hastie, and T.C. Hender, Phys. Plasmas 3, 3369 (1996).

${ }^{20}$ F. Porcelli, R. Stankiewicz, W. Kerner, and H.L. Berk, Phys. Plasmas 1, 470 (1994). 


\section{External Distribution}

Plasma Research Laboratory, Australian National University, Australia

Professor I.R. Jones, Flinders University, Australia

Professor João Canalle, Instituto de Fisica DEQ/IF - UERJ, Brazil

Mr. Gerson O. Ludwig, Instituto Nacional de Pesquisas, Brazil

Dr. P.H. Sakanaka, Instituto Fisica, Brazil

The Librarian, Culham Laboratory, England

Mrs. S.A. Hutchinson, JET Library, England

Professor M.N. Bussac, Ecole Polytechnique, France

Librarian, Max-Planck-Institut für Plasmaphysik, Germany

Jolan Moldvai, Reports Library, MTA KFKI-ATKI, Hungary

Dr. P. Kaw, Institute for Plasma Research, India

Ms. P.J. Pathak, Librarian, Insitute for Plasma Research, India

Ms. Clelia De Palo, Associazione EURATOM-ENEA, Italy

Dr. G. Grosso, Instituto di Fisica del Plasma, Italy

Librarian, Naka Fusion Research Establishment, JAERI, Japan

Library, Plasma Physics Laboratory, Kyoto University, Japan

Research Information Center, National Institute for Fusion Science, Japan

Dr. O. Mitarai, Kyushu Tokai University, Japan

Library, Academia Sinica, Institute of Plasma Physics, People's Republic of China

Shih-Tung Tsai, Institute of Physics, Chinese Academy of Sciences, People's Republic of China

Dr. S. Mirnov, TRINITI, Troitsk, Russian Federation, Russia

Dr. V.S. Strelkov, Kurchatov Institute, Russian Federation, Russia

Professor Peter Lukac, Katedra Fyziky Plazmy MFF UK, Mlynska dolina F-2, Komenskeho Univerzita, SK-842 15 Bratislava, Slovakia

Dr. G.S. Lee, Korea Basic Science Institute, South Korea

Institute for Plasma Research, University of Maryland, USA

Librarian, Fusion Energy Division, Oak Ridge National Laboratory, USA

Librarian, Institute of Fusion Studies, University of Texas, USA

Librarian, Magnetic Fusion Program, Lawrence Livermore National Laboratory, USA

Library, General Atomics, USA

Plasma Physics Group, Fusion Energy Research Program, University of California at San Diego, USA

Plasma Physics Library, Columbia University, USA

Alkesh Punjabi, Center for Fusion Research and Training, Hampton University, USA

Dr. W.M. Stacey, Fusion Research Center, Georgia Institute of Technology, USA

Dr. John Willis, U.S. Department of Energy, Office of Fusion Energy Sciences, USA

Mr. Paul H. Wright, Indianapolis, Indiana, USA 
The Princeton Plasma Physics Laboratory is operated by Princeton University under contract with the U.S. Department of Energy.

\author{
Information Services \\ Princeton Plasma Physics Laboratory \\ P.O. Box 451 \\ Princeton, NJ 08543
}

Phone: 609-243-2750

Fax: 609-243-2751

e-mail: pppl_info@pppl.gov

Internet Address: http://www.pppl.gov 